\title{
The most cited authors and papers in tobacco control
}

\section{F Byrne, S Chapman}

Tobacco Control 2005;14:155-160. doi: 10.1136/tc.2005.011973

w this paper, we present the first attempt at determining which authors of research and commentary of direct relevance to tobacco control have the most cited publications in this field. We examine this from 1980 to 2004 and also for the past decade (1994-2004) in an effort to distinguish the 100 overall most cited authors for these periods. We have also provided a list of the 50 highest citation classics in tobacco control.

Citations are the most common way of measuring the impact in the scientific community of an article, and cumulatively, of a researcher. ${ }^{1-3}$ However, there can be important differences between evaluating impact and the quality of a paper. The quality of a paper is essentially characterised by the notion of possible value and this cannot be easily measured in an objective and quantitative way. ${ }^{4}$ Smith has suggested a range of ways in which scientific output might otherwise be evaluated, but all of these outcomes can be less easily measured than by calculating citations. ${ }^{5}$ While use of databases for citation analysis have been widely criticised, ${ }^{6}$ they do offer the easiest approach for assembling "ball park" data even when taking into consideration that the databases do not distinguish between positive and negative credits. Authors generally cite exemplary or important articles. While controversial badly flawed articles can also be highly cited in critical articles referring to them, it seldom happens that an author will repeatedly produce such highly cited flawed articles.

The field of tobacco control is very broad and includes basic science on toxicology, pharmacology, and genetics; clinical research reporting on the impact of tobacco use on individuals' health; epidemiological research on large populations; studies of tobacco use; and matters relevant to policies and programmes designed to reduce tobacco use and the harm it causes. There is a huge body of epidemiological research, which examines multiple risk factors for disease, where tobacco use is only one variable examined. Such research can be vital to the evidence base that underlies the case for tobacco control and the content of public awareness campaigns. As will be seen, many authors of such studies have been ranked highly and dominate the lists. While some may question their inclusion arguing that multiple risk factor epidemiology is not "true" tobacco control research, we reject that argument on both substantive and practical grounds. Culling such articles manually would have taken hundreds of hours, generated understandable controversy, and resulted in many highly influential epidemiological studies being capriciously discarded for the dubious purpose of elevating some authors doing more work precisely focused around tobacco "control".

\section{METHODS}

Authors were identified using the Thomson Institute of Scientific Information (ISI) Web of Science (WoS) database. WoS includes original contributions, reviews, letters, editorials, text chapters, and published conference abstracts. None of these were excluded, on the rationale that any type of paper that was highly cited was of interest to the field. In August 2004, advanced WoS search queries combining topic and title fields were conducted to facilitate the construction of a shortlist of authors. The search string TS $=\left(\right.$ tobacco $^{*} \mathrm{OR}$ smok $^{*}$ OR cigarette* OR nicotine* OR nonsmok ${ }^{*}$ ) OR $\mathrm{TI}=\left(\right.$ tobacco $^{*}$ OR smok ${ }^{*}$ OR cigarette* OR nicotine* ${ }^{*}$ OR nonsmok ${ }^{*} \dagger$ was run for each year between 1980 to 2004 . The search covered all articles indexed in the Science Citation Index Expanded, the Social Science Citation Index, and the Arts and Humanities Citation Index, and 106789 records were downloaded.

As WoS has a download limit of 500 citations, the data were downloaded in batches of 500 for each year. The accumulated batches were then imported into two databases: Histcite Trial Version (HistCite (Vlad) Version: 2004.05.14) and Postgres SQL. Histcite is a system designed to assist in identifying the most cited papers retrieved in topical searches of WoS. ${ }^{7}$ HistCite can create tables by author, year, or citation frequency. ${ }^{7}$

The data were imported into HistCite in annual batches and a bibliography representing each year was saved. The bibliographies were then re-imported into HistCite and merged to a level at which the database would cope: 19801989, 1990-1996, 1997-1999, 2000-2001 and 2002-2004.

To generate the shortlist of authors for 1980-2004, the top 300 names by citation frequency from each batch were then extracted and imported into Excel, subsequently merged, and re-sorted by citation frequency. Names and associated papers were reviewed by revisiting the complete dataset in the Postgres SQL database. Importantly, to appear on the shortlist of authors, a person needed to have published at least 10 publications as first author.

The same procedure was carried out to compile a list of authors for the period 1994-2004.

Updated data for the authors on the shortlists were collected in February 2005. As the WoS database is updated on a weekly basis, the top 100 names for each time period were searched individually and combined with the following topic/title search using WoS data updated as at 12 February 2005:

$\mathrm{TS}=\left(\mathrm{TS}=\left(\right.\right.$ tobacco $^{*} \mathrm{OR}$ smok $^{*}$ OR cigarette ${ }^{*}$ OR nicotine $\mathrm{OR}^{*} \mathrm{OR}$ nonsmok $\left.{ }^{*}\right)$ OR TI $=\left(\right.$ tobacco* OR smok $^{*}$ OR cigarette ${ }^{*}$ OR nicotine $^{*}$ OR nonsmok $\left.\left.{ }^{*}\right)\right)$ AND AU $=\left(\left(\mathrm{AU}=\right.\right.$ Surname Initial $\left.\left.{ }^{*}\right)\right)$ The settings were DocType $=$ All document types; Language $=$ All languages; Databases $=$ SCI-EXPANDED, SSCI, A\&HCI; Timespan $=$ The specified time period which was either 1994-2004 OR 1980-2004.

The downloaded data were then imported into HistCite as well as an Endnote 7.0 Library for each dataset (1980-2004 and 1994-2004). HistCite was used to compute the preliminary data and the results were exported into two Excel workbooks (1980-2004 and 1994-2004). Each name was cross checked using the data in the Endnote Library to detect any anomalies and also to confirm that each author had published $\geqslant 10$ papers as first author. ${ }^{\dagger}$ A list of exclusion keywords was run in conjunction with the topic
search to primarily exclude results concerned with tobacco agronomy. 
Table 1 Top 100 authors 1980-2004

\begin{tabular}{|c|c|c|c|c|c|c|c|c|}
\hline Rank & Author & Sex & Country & Total cites & Total papers & Cites/paper & First author & Field* \\
\hline 1 & Stampfer MJ & $M$ & USA & 27819 & 235 & 118.4 & 10 & 2 \\
\hline 2 & Willett WC & $M$ & USA & 24601 & 279 & 88.2 & 13 & 2 \\
\hline 3 & Colditz GA & $M$ & USA & 15983 & 201 & 79.5 & 19 & 2 \\
\hline 4 & Ridker PM & $M$ & USA & 10148 & 78 & 130.1 & 34 & 2 \\
\hline 5 & Prochaska JO & $M$ & USA & 8521 & 86 & 99.1 & 30 & 8 \\
\hline 6 & Benowitz NL & $M$ & USA & 8164 & 301 & 27.1 & 104 & 7 \\
\hline 7 & Wilson PWF & $M$ & USA & 7321 & 113 & 64.8 & 16 & 2 \\
\hline 8 & Giovannucci E & $M$ & USA & 6498 & 87 & 74.7 & 24 & 2 \\
\hline 9 & Hecht SS & $M$ & USA & 6269 & 217 & 28.9 & 80 & 11 \\
\hline 10 & DiClemente CC & $M$ & USA & 6051 & 51 & 118.7 & 11 & 8 \\
\hline 11 & La Vecchia C & $M$ & Italy & 5788 & 305 & 19.0 & 42 & 2 \\
\hline 12 & Folsom AR & $M$ & USA & 5523 & 140 & 39.5 & 17 & 2 \\
\hline 13 & Hoffmann D & $M$ & USA & 5495 & 184 & 29.9 & 45 & 11 \\
\hline 14 & Collins AC & $M$ & USA & 5303 & 147 & 36.1 & 16 & 5 \\
\hline 15 & Salonen JT & M & Finland & 5147 & 97 & 53.1 & 22 & 2 \\
\hline 16 & Barrett-Connor E & $\mathrm{F}$ & USA & 5018 & 103 & 48.7 & 22 & 2 \\
\hline 17 & Kannel WB & $M$ & USA & 4985 & 69 & 72.3 & 29 & 2 \\
\hline 18 & Weiss ST & $M$ & USA & 4931 & 169 & 29.2 & 18 & 4 \\
\hline 19 & Kromhout D & M & Netherlands & 4796 & 112 & 42.8 & 11 & 2 \\
\hline 20 & Russell MAH & $M$ & UK & 4558 & 114 & 40.0 & 34 & 5 \\
\hline 21 & Thun MJ & $M$ & USA & 4457 & 91 & 49.0 & 15 & 2 \\
\hline 22 & Velicer WF & $M$ & USA & 4416 & 61 & 72.4 & 13 & 8 \\
\hline 23 & Peto R & $M$ & UK & 4311 & 56 & 77.0 & 18 & 2 \\
\hline 24 & Hughes JR & M & USA & 4131 & 148 & 27.9 & 100 & 5 \\
\hline 25 & Jacob P & $M$ & USA & 4127 & 126 & 32.8 & 24 & 7 \\
\hline 26 & Tuomilehto J & $M$ & Finland & 4079 & 112 & 36.4 & 13 & 2 \\
\hline 27 & Pierce JP & $M$ & USA & 4060 & 129 & 31.5 & 44 & 2 \\
\hline 28 & Jarvis $M$ & $M$ & UK & 3941 & 128 & 30.8 & 58 & 8 \\
\hline 29 & Friedman GD & M & USA & 3910 & 60 & 65.2 & 19 & 2 \\
\hline 30 & Fiore MC & M & USA & 3879 & 100 & 38.8 & 32 & 4 \\
\hline 31 & Marks MJ & M & USA & 3773 & 88 & 42.9 & 27 & 3 \\
\hline 32 & McLaughlin JK & M & USA & 3729 & 101 & 36.9 & 14 & 4 \\
\hline 33 & Franceschi S & $\mathrm{F}$ & Italy & 3660 & 171 & 21.4 & 23 & 2 \\
\hline 34 & Kuller LH & $M$ & USA & 3655 & 98 & 37.3 & 16 & 2 \\
\hline 35 & Clarke PBS & $M$ & UK/Canada & 3530 & 52 & 67.9 & 28 & 5 \\
\hline 36 & Stamler J & $M$ & USA & 3526 & 60 & 58.8 & 10 & 4 \\
\hline 37 & Henningfield JE & $M$ & USA & 3521 & 167 & 21.1 & 64 & 5 \\
\hline 38 & Negri $\mathrm{E}$ & $\mathrm{F}$ & Italy & 3495 & 179 & 19.5 & 19 & 2 \\
\hline 39 & Lichtenstein $\mathrm{E}$ & $M$ & USA & 3478 & 101 & 34.4 & 20 & 8 \\
\hline 40 & Wynder EL & $M$ & USA & 3468 & 129 & 26.9 & 30 & 2 \\
\hline 41 & $\mathrm{Hu} F B$ & M & USA & 3306 & 70 & 47.2 & 29 & 2 \\
\hline 42 & Flay BR & $M$ & USA & 3302 & 102 & 32.4 & 14 & 8 \\
\hline 43 & Bartsch H & $M$ & Germany & 3288 & 95 & 34.6 & 14 & 11 \\
\hline 44 & Smith GD & $M$ & UK & 3271 & 117 & 28.0 & 22 & 2 \\
\hline 45 & Strachan DP & $M$ & UK & 3239 & 64 & 50.6 & 23 & 2 \\
\hline 46 & Spitz MR & $\mathrm{F}$ & USA & 3224 & 134 & 24.1 & 18 & 2 \\
\hline 47 & Levin ED & M & USA & 3062 & 104 & 29.4 & 57 & 5 \\
\hline 48 & Nordberg A & $\mathrm{F}$ & Sweden & 3020 & 98 & 30.8 & 24 & 5 \\
\hline 49 & Martinez FD & $M$ & USA & 2985 & 40 & 74.6 & 10 & 4 \\
\hline 50 & Tashkin DP & M & USA & 2957 & 120 & 24.6 & 33 & 4 \\
\hline 51 & Abrams DB & $M$ & USA & 2874 & 110 & 26.1 & 11 & 8 \\
\hline 52 & Pryor WA & $M$ & USA & 2837 & 68 & 41.7 & 19 & 1 \\
\hline 53 & Yu MC & $\mathrm{F}$ & USA & 2767 & 71 & 39.0 & 13 & 2 \\
\hline 54 & Vineis $P$ & $M$ & Italy & 2746 & 91 & 30.2 & 32 & 2 \\
\hline 55 & Fortmann SP & M & USA & 2741 & 63 & 43.5 & 11 & 2 \\
\hline 56 & Lowe GDO & M & UK & 2675 & 67 & 39.9 & 12 & 4 \\
\hline 57 & Marcus BH & $\mathrm{F}$ & USA & 2657 & 50 & 53.1 & 20 & 8 \\
\hline 58 & Trichopoulos D & $M$ & Greece/USA & 2617 & 97 & 27.0 & 12 & 2 \\
\hline 59 & Murray DM & $M$ & USA & 2611 & 75 & 34.8 & 23 & 8 \\
\hline 60 & Slotkin TA & M & USA & 2608 & 94 & 27.7 & 28 & 7 \\
\hline 61 & Knekt P & M & Finland & 2601 & 51 & 51.0 & 22 & 2 \\
\hline 62 & Ockene JK & $\mathrm{F}$ & USA & 2592 & 99 & 26.2 & 31 & 2 \\
\hline 63 & $X u X P$ & $M$ & USA & 2579 & 66 & 39.1 & 21 & 2 \\
\hline 64 & Hurt RD & $M$ & USA & 2563 & 133 & 19.3 & 37 & 4 \\
\hline 65 & Perry CL & $\mathrm{F}$ & USA & 2561 & 55 & 46.6 & 18 & 9 \\
\hline 66 & Barnes PJ & $M$ & UK & 2559 & 90 & 28.4 & 13 & 4 \\
\hline 67 & Glassman $\mathrm{AH}$ & $M$ & USA & 2526 & 29 & 87.1 & 14 & 5 \\
\hline 68 & Doll R & $M$ & UK & 2497 & 42 & 59.5 & 25 & 2 \\
\hline 69 & Klein R & $M$ & USA & 2441 & 95 & 25.7 & 26 & 6 \\
\hline 70 & Glantz SA & $M$ & USA & 2431 & 167 & 14.6 & 51 & 10 \\
\hline 71 & Rose JE & $M$ & USA & 2426 & 109 & 22.3 & 44 & 5 \\
\hline 72 & Glasgow RE & M & USA & 2357 & 95 & 24.8 & 38 & 8 \\
\hline 73 & Lebowitz MD & M & USA & 2313 & 70 & 33.0 & 19 & 2 \\
\hline 74 & Samet JM & M & USA & 2302 & 94 & 24.5 & 29 & 2 \\
\hline 75 & Shiffman S & $M$ & USA & 2271 & 98 & 23.2 & 53 & 5 \\
\hline 76 & Rennard SI & M & USA & 2243 & 97 & 23.1 & 15 & 4 \\
\hline 77 & Klein BEK & $\mathrm{F}$ & USA & 2232 & 81 & 27.6 & 12 & 6 \\
\hline 78 & Shaper AG & $M$ & UK & 2229 & 61 & 36.5 & 10 & 2 \\
\hline 79 & Boffetta P & $M$ & France/Germany & 2228 & 147 & 15.2 & 30 & 2 \\
\hline 80 & Kozlowski LT & $\mathrm{F}$ & Canada/USA & 2197 & 95 & 23.1 & 66 & 8 \\
\hline 81 & Baron JA & $M$ & USA & 2184 & 62 & 35.2 & 24 & 2 \\
\hline
\end{tabular}




\begin{tabular}{|c|c|c|c|c|c|c|c|c|}
\hline Rank & Author & Sex & Country & Total cites & Total papers & Cites/paper & First author & Field* \\
\hline 82 & Hogg JC & $M$ & Canada & 2167 & 84 & 25.8 & 13 & 4 \\
\hline 83 & Cummings SR & M & USA & 2144 & 44 & 48.7 & 10 & 2 \\
\hline 84 & Perera FP & $\mathrm{F}$ & USA & 2133 & 62 & 34.4 & 19 & 2 \\
\hline 85 & Schwartz J & M & USA & 2096 & 47 & 44.6 & 13 & 2 \\
\hline 86 & Tager IB & M & USA & 2081 & 50 & 41.6 & 16 & 2 \\
\hline 87 & Omenn GS & M & USA & 2077 & 29 & 71.6 & 12 & 2 \\
\hline 88 & Vartiainen $\mathrm{E}$ & M & Finland & 2000 & 77 & 26.0 & 16 & 2 \\
\hline 89 & Gilpin EA & $\mathrm{F}$ & USA & 1981 & 69 & 28.7 & 20 & 10 \\
\hline 90 & Luepker RV & M & USA & 1976 & 50 & 39.5 & 10 & 2 \\
\hline 91 & Wonnacott S & $\mathrm{F}$ & UK & 1968 & 64 & 30.8 & 12 & 5 \\
\hline 92 & Hemminki K & M & Sweden & 1940 & 79 & 24.6 & 22 & 2 \\
\hline 93 & Botvin GJ & M & USA & 1919 & 81 & 23.7 & 27 & 8 \\
\hline 94 & Stolerman IP & M & UK & 1902 & 96 & 19.8 & 36 & 5 \\
\hline 95 & Vainio $\mathrm{H}$ & M & Finland/France/Sweden & 1896 & 74 & 25.6 & 17 & 4 \\
\hline 96 & Fagerstrom $\mathrm{KO}$ & M & Sweden & 1884 & 49 & 38.5 & 30 & 8 \\
\hline 97 & Jorenby DE & M & USA & 1876 & 42 & 44.7 & 11 & 8 \\
\hline 98 & Boyle P & M & Italy & 1859 & 96 & 19.4 & 18 & 2 \\
\hline 99 & Orleans CT & $\mathrm{F}$ & USA & 1855 & 71 & 26.1 & 26 & 8 \\
\hline 100 & Hall SM & $\mathrm{F}$ & USA & 1847 & 53 & 34.9 & 20 & 8 \\
\hline
\end{tabular}

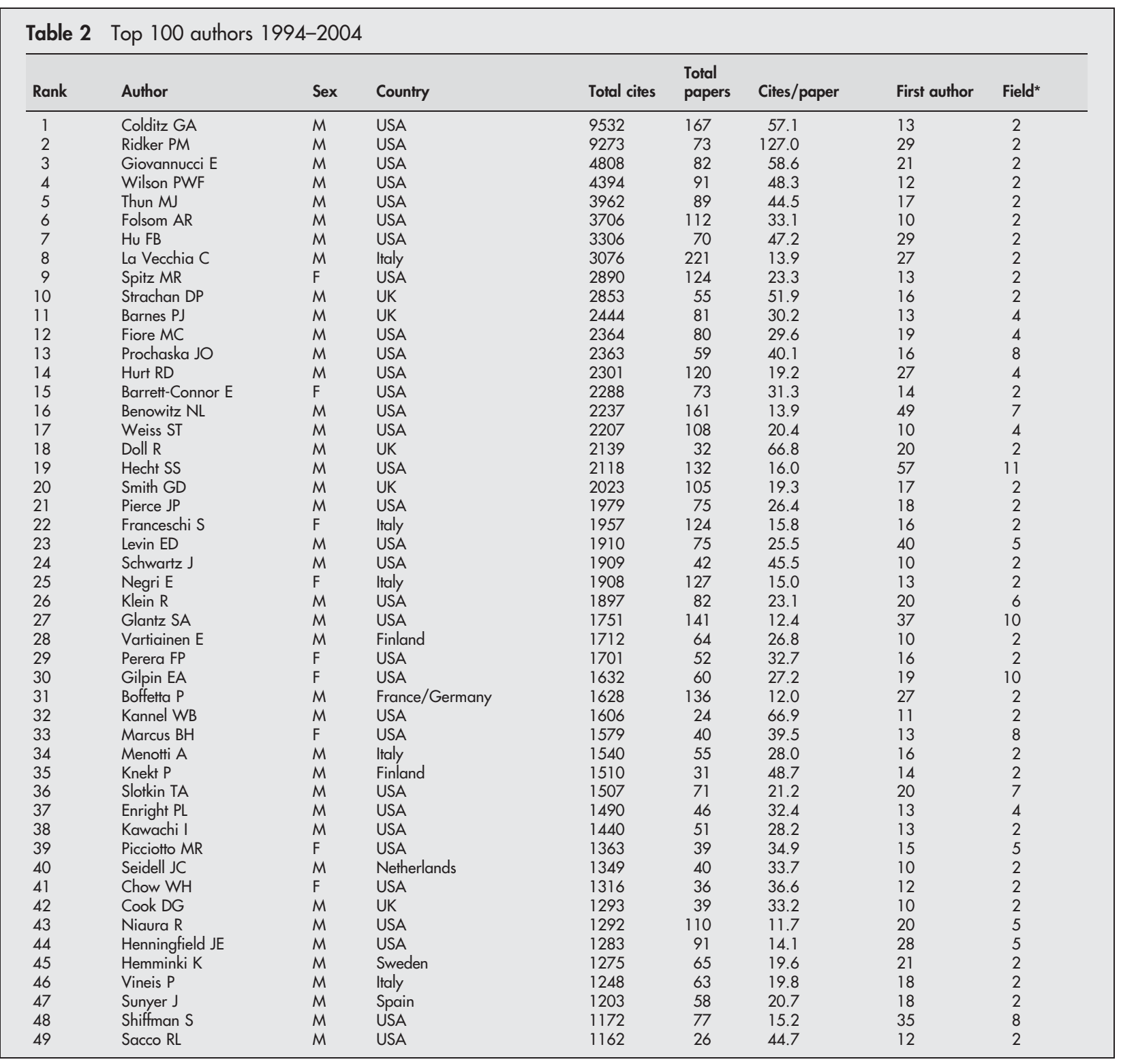




\begin{tabular}{|c|c|c|c|c|c|c|c|c|}
\hline Rank & Author & Sex & Country & Total cites & $\begin{array}{l}\text { Total } \\
\text { papers }\end{array}$ & Cites/paper & First author & Field $^{*}$ \\
\hline 50 & Wu XF & $\mathrm{F}$ & USA & 1151 & 60 & 19.2 & 18 & 2 \\
\hline 51 & Samet JM & M & USA & 1148 & 52 & 22.1 & 15 & 2 \\
\hline 52 & Levi F & M & Switzerland & 1145 & 80 & 14.3 & 33 & 2 \\
\hline 53 & Emmons KM & $\mathrm{F}$ & USA & 1137 & 67 & 17.0 & 22 & 8 \\
\hline 54 & Law MR & M & UK & 1136 & 20 & 56.8 & 14 & 2 \\
\hline 55 & Rose JE & M & USA & 1115 & 53 & 21.0 & 23 & 5 \\
\hline 56 & Howard G & M & USA & 1047 & 26 & 40.3 & 10 & 10 \\
\hline 57 & Jousilahti P & M & Finland & 1030 & 36 & 28.6 & 19 & 2 \\
\hline 58 & Vainio $\mathrm{H}$ & M & Finland/France/Sweden & 1028 & 51 & 20.2 & 12 & 4 \\
\hline 59 & Boyle P & M & Italy & 1017 & 67 & 15.2 & 16 & 2 \\
\hline 60 & Benhamou S & $\mathrm{F}$ & France & 1013 & 50 & 20.3 & 13 & 10 \\
\hline 61 & Glasgow RE & M & USA & 1011 & 55 & 18.4 & 18 & 8 \\
\hline 62 & Murray DM & M & USA & 974 & 45 & 21.6 & 11 & 8 \\
\hline 63 & Rigotti NA & $\mathrm{F}$ & USA & 950 & 70 & 13.6 & 21 & 4 \\
\hline 64 & Saetta $M$ & $\mathrm{~F}$ & Italy & 945 & 34 & 27.8 & 11 & 4 \\
\hline 65 & Hart CL & $\mathrm{F}$ & UK & 940 & 44 & 21.4 & 13 & 10 \\
\hline 66 & Wynder EL & M & USA & 934 & 47 & 19.9 & 11 & 2 \\
\hline 67 & Xu XP & M & USA & 927 & 57 & 16.3 & 14 & 2 \\
\hline 68 & Jacob P & M & USA & 906 & 67 & 13.5 & 12 & 1 \\
\hline 69 & Cerhan JR & M & USA & 902 & 45 & 20.0 & 13 & 2 \\
\hline 70 & Rahman I & M & UK & 901 & 32 & 28.2 & 18 & 4 \\
\hline 71 & Britton J & $M$ & UK & 885 & 52 & 17.0 & 21 & 4 \\
\hline 72 & Perkins KA & M & USA & 866 & 77 & 11.2 & 55 & 8 \\
\hline 73 & Nakajima M & $M$ & Japan & 858 & 36 & 23.8 & 19 & 7 \\
\hline 74 & Lichtenstein $E$ & M & USA & 852 & 55 & 15.5 & 11 & 8 \\
\hline 75 & Baron JA & $M$ & USA & 846 & 34 & 24.9 & 13 & 2 \\
\hline 76 & Hughes JR & M & USA & 843 & 82 & 10.3 & 56 & 5 \\
\hline 77 & Ford ES & $M$ & USA & 826 & 45 & 18.4 & 23 & 2 \\
\hline 78 & Ritz E & M & Germany & 816 & 32 & 25.5 & 11 & 4 \\
\hline 79 & Stolerman IP & M & UK & 805 & 59 & 13.6 & 18 & 2 \\
\hline 80 & Brownson RC & M & USA & 805 & 45 & 17.9 & 12 & 5 \\
\hline 81 & Wetter DW & M & USA & 803 & 32 & 25.1 & 11 & 8 \\
\hline 82 & Orleans CT & $\mathrm{F}$ & USA & 801 & 46 & 17.4 & 13 & 8 \\
\hline 83 & Mancia G & M & Italy & 789 & 31 & 25.5 & 10 & 4 \\
\hline 84 & Prescott E & $\mathrm{F}$ & Denmark & 789 & 49 & 16.1 & 21 & 4 \\
\hline 85 & Damaj MI & M & USA & 787 & 43 & 18.3 & 21 & 7 \\
\hline 86 & Botvin GJ & M & USA & 781 & 56 & 13.9 & 13 & 8 \\
\hline 87 & Breslau N & $\mathrm{F}$ & USA & 772 & 30 & 25.7 & 18 & 2 \\
\hline 88 & Sussman $\mathrm{S}$ & M & USA & 753 & 61 & 12.3 & 26 & 8 \\
\hline 89 & Kandel DB & $\mathrm{F}$ & USA & 744 & 18 & 41.3 & 10 & 2 \\
\hline 90 & Tavani A & $\mathrm{F}$ & Italy & 742 & 48 & 15.5 & 31 & 2 \\
\hline 91 & Staessen JA & M & Belgium & 738 & 26 & 28.4 & 10 & 4 \\
\hline 92 & Nyberg F & M & Sweden & 714 & 49 & 14.6 & 11 & 2 \\
\hline 93 & Cnattingius $S$ & M & Sweden & 712 & 44 & 16.2 & 12 & 2 \\
\hline 94 & Sorensen G & $\mathrm{F}$ & USA & 690 & 39 & 17.7 & 19 & 9 \\
\hline 95 & Inoue $M$ & $\mathrm{~F}$ & Japan & 682 & 48 & 14.2 & 14 & 2 \\
\hline 96 & Zhang ZF & M & USA & 661 & 49 & 13.5 & 12 & 2 \\
\hline 97 & Jeffery RW & M & USA & 660 & 30 & 22.0 & 10 & 8 \\
\hline 98 & DiFranza JR & M & USA & 659 & 41 & 16.1 & 27 & 4 \\
\hline 99 & Lam S & M & Canada & 657 & 18 & 36.5 & 10 & 4 \\
\hline 100 & DuRant RH & M & USA & 636 & 28 & 22.7 & 13 & 4 \\
\hline
\end{tabular}

\begin{tabular}{|c|c|c|}
\hline \multicolumn{3}{|c|}{ Field key } \\
\hline Code & Field & Notes \\
\hline 1 & Chemistry & Includes biochemistry \\
\hline 2 & Epidemiólogy & \\
\hline 3 & Genetics & \\
\hline 4 & Medicine & $\begin{array}{l}\text { All branches of medicine, including } \\
\text { family medicine, internal medicine, } \\
\text { thoracic medicine, preventive } \\
\text { medicine, adolescent medicine and } \\
\text { paediatrics }\end{array}$ \\
\hline 5 & Neuropsychopharmacology & $\begin{array}{l}\text { Includes neuropsychology, } \\
\text { psychopharmacology, behavioural } \\
\text { pharmacology }\end{array}$ \\
\hline 6 & Ophthalmology & \\
\hline 7 & Pharmacology & \\
\hline 8 & Psychology & $\begin{array}{l}\text { Includes all branches, includes } \\
\text { clinical psychology }\end{array}$ \\
\hline 9 & Sociology/education & \\
\hline 10 & Statistics & Includes biostatistics \\
\hline 11 & $\begin{array}{l}\text { Tobacco carcinogenesis/ } \\
\text { environmental carcinogenesis }\end{array}$ & \\
\hline
\end{tabular}

The top 50 articles were selected from the initial August 2004 data download. Papers that had more than 150 citations (957 in total) were exported from the Postgres SQL database and imported into an Excel spreadsheet for sorting and analysis. Papers were selected if they were on tobacco use prevention and control as well as epidemiological papers regarded as seminal in establishing the harms of tobacco use.

\section{RESULTS}

Our final analysis included 9745 papers for the period 19802004 and 7644 papers for the period 1994-2004. Table 1 shows the 100 most cited individuals 1980-2004 by total citations. The honour of the most cited author goes to Meir Stampfer from the Harvard School of Public Health. The person who has the highest mean number of citations is Paul Ridker, Eugene Braunwald Professor of Medicine at Harvard Medical School. Over 70\% of authors are from North America with the remainder from Europe. There are a higher percentage of male authors (84\%) than female authors $(16 \%)$. Forty seven per cent of the authors conduct 
Table 3 Top 50 papers selected as citation classics (August 2004 download data)

$\begin{array}{ll}\text { Rank Paper } & \text { Times } \\ \text { cited }\end{array}$

1 Prochaska JO, Diclemente CC, Norcross JC. In search of how people change: applications to addictive behaviors Am Psychol 1992;47:1102-14.

2 Prochaska JO, Diclemente CC. Stages and processes of self-change of smoking: toward an integrative model of change. J Consult Clin Psychol 1983;51:390-95

3 McGinnis JM, Foege WH. Actual causes of death in the United States. JAMA 1993;270:2207-12.

4 Davis DA, Thomson MA, Oxman AD, et al. Changing physician performance: a systematic review of the effect of continuing medical education strategies. JAMA 1995:274:700-05.

5 Hjermann I, Holme I, Byre KV, et al. Effect of diet and smoking intervention on the incidence of coronary heart disease: report from the Oslo Study Group of a randomized trial in healthy men. Lancet 1981:2:1303-10.

6 Doll R, Peto R, Wheatley K, et al. Mortality in relation to smoking: 40 years' observations on male British doctors. BMJ 1994;309:901-11.

7 Heatherton TF, Kozlowski LT, Frecker RC, et al. The Fagerstrom Test for Nicotine Dependence: a revision of the Fagerstrom Tolerance Questionnaire. Br J Addict 1991;86:1119-27.

8 Couturier S, Bertrand D, Matter JM, et al. A neuronal nicotinic acetylcholine receptor subunit (alpha-7) is developmentally regulated and forms a homo-oligomeric channel blocked by alpha-BTX. Neuron 1990;5:847-56.

9 Diclemente CC, Fairhurst SK, Velasquez MM, et al. The process of smoking cessation: an analysis of precontemplation, contemplation, and preparation stages of change. J Consult Clin Psychol 1991;59:295-304.

10 Resnick MD, Bearman PS, Blum RW, et al. Protecting adolescents from harm: findings from the national longitudinal study on adolescent health. JAMA 1997;278:823-32.

11 Celermajer DS, Sorensen KE, Georgakopoulos D, et al. Cigarette smoking is associated with dose-related and potentially reversible impairment of endotheliumdependent dilation in healthy-young adults. Circulation 1993:88:2149-55.

12 Lundberg JM, Saria A. Capsaicin-induced desensitization of airway mucosa to cigarette-smoke, mechanical and chemical irritants. Nature 1983;302:251-53.

13 Flores CM, Rogers SW, Pabreza LA, et al. A subtype of nicotinic cholinergic receptor in rat-brain is composed of alpha- 4 and beta- 2 subunits and is up-regulated by chronic nicotine treatment. Mol Pharmaco 1992;41:31-37

14 Prochaska JO, Velicer WF, Rossi JS, et al. Stages of change and decisional balance for 12 problem behaviors. Health Psychol 1994;13:39-46.

15 Benowitz NL. Pharmacologic aspects of cigarette smoking and nicotine addiction. N Engl J Med 1988;319:1318-30.

16 McGehee DS, Heath MJS, Gelber S, et al. Nicotine enhancement of fast excitatory synaptic transmission in CNS by presynaptic receptors. Science 1995;269: 1692-96.

17 Ames BN, Gold LS, Willett WC. The causes and prevention of cancer. Proc Natl Acad Sci USA 1995;92:5258-65.

18 Church DF, Pryor WA. Free-radical chemistry of cigarette smoke and its toxicological implications. Environ Health Perspect 1985;64:111-26.

19 Murray CJL, Lopez AD. Global mortality, disability, and the contribution of risk factors: Global Burden of Disease Study. Lancet 1997:349:1436-42.

20 Peto R, Lopez AD, Boreham J, et al. Mortality from tobacco in developed-countries: indirect estimation from national vital-statistics. Lancet 1992;339:1268-78.

21 Leventhal H, Cleary PD. The smoking problem: a review of the research and theory in behavioral risk modification. Psychol Bull 1980;88:370-405.

22 Anthonisen NR, Connett JE, Kiley JP, et al. Effects of smoking intervention and the use of an inhaled anticholinergic bronchodilator on the rate of decline of FEV 1 : the Lung Health Study. JAMA 1994; 272:1497-505.

23 Oxman AD, Thomson MA, Davis DA, et al. No magic bullets: a systematic review of 102 trials of interventions to improve professional practice. CMAJ $1995 ; 153: 1423-31$
Table 3 Continued

Rank Paper Times
cited

24 Wilson PWF, Garrison RJ, Castelli WP. Postmenopausal estrogen use, cigarette-smoking, and cardiovascular morbidity in women over 50: the Framingham Study. N Engl J Med 1985;313:1038-43.

25 Glassman AH, Helzer JE, Covey LS, et al. Smoking, smoking cessation, and major depression. JAMA 1990;264: 1546-49

26 Hirayama T. Non-smoking wives of heavy smokers have a higher risk of lung-cancer: a study from Japan. Br Med J (Clin Res Ed) 1981;282:183-85.

27 Blot WJ, McLaughlin JK, Winn DM, et al. Smoking and drinking in relation to oral and pharyngeal cancer. Cancer Res 1988;48:3282-87.

28 Imperato A, Mulas A, Dichiara G. Nicotine preferentially stimulates dopamine release in the limbic system of freely moving rats. Eur J Pharmacol 1986; 132:337-38.

29 Hecht SS, Hoffmann D. Tobacco-specific nitrosamines, an important group of carcinogens in tobacco and tobacco-smoke. Carcinogenesis 1988:9:875-84.

30 Hurt RD, Sachs DPL, Glover ED, et al. A comparison of sustained-release bupropion and placebo for smoking cessation. N Engl J Med 1997;337:1 195-202.

31 Hughes JR, Hatsukami DK, Mitchell JE, et al. Prevalence of smoking among psychiatric outpatients. Am J Psychiatry 1986;143:993-97.

32 Fagerstrom KO, Schneider NG. Measuring nicotine dependence:- a review of the Fagerstrom Tolerance Questionnaire. J Behav Med 1989;12:159-82.

33 Hughes JR, Hatsukami D. Signs and symptoms of tobacco withdrawal. Arch Gen Psychiatry 1986;43:289-94

34 Jorenby DE, Leischow SJ, Nides MA, et al. A controlled trial of sustained-release bupropion, a nicotine patch, or both for smoking cessation. N Engl J Med 1999;340:685-91.

35 Hunninghake GW, Crystal RG. Cigarette-smoking and lung destruction: accumulation of neutrophils in the lungs of cigarette smokers. Am Rev Respir Dis 1983; 128:833-38.

36 Anda RF, Williamson DF, Escobedo LG, et al. Depression and the dynamics of smoking: a national perspective. JAMA 1990;264:1541-45.

37 Levin ED. Nicotinic systems and cognitive function. Psychopharmacology 1992;108:417-31.

38 Condiotte MM, Lichtenstein E. Self-efficacy and relapse in smoking cessation programs. J Consult Clin Psychol 1981;49:648-58

39 Jones JG, Lawler P, Crawley JCW, et al. Increased alveolar epithelial permeability in cigarette smokers. Lancet 1980;1:66-68.

40 Kannel WB, Belanger AJ. Epidemiology of heart failure. Am Heart J 1991;121:951-57.

41 Stryker WS, Kaplan LA, Stein EA, et al. The relation of diet, cigarette-smoking, and alcohol-consumption to plasma beta-carotene and alpha-tocopherol levels. Am J Epidemiol 1988;127:283-96.

42 Shinton R, Beevers G. Meta-analysis of relation between cigarette smoking and stroke. BMJ 1989;298:789-94.

43 Shiffman S. Relapse following smoking cessation: a situational analysis. J Consult Clin Psychol 1982;50:71-86.

44 Hoffmann D, Hecht SS. Nicotine-derived N-nitrosamines and tobacco-related cancer: current status and future directions. Cancer Res 1985;45:935-44.

45 Jarvis MJ, Tunstall Pedoe H, Feyerabend $C$, et al. Comparison of tests used to distinguish smokers from nonsmokers. Am J Public Health 1987:77:1435-38.

46 Meade TW, Imeson J, Stirling Y. Effects of changes in smoking and other characteristics on clotting factors and the risk of ischemic heart disease. Lancet 1987;2:986-88.

47 Celermajer DS, Adams MR, Clarkson P, et al. Passive smoking and impaired endothelium-dependent arterial dilatation in healthy young adults. N Engl I Med 1996;334:150-54.

48 Pontieri FE, Tanda G, Orzi F, et al. Effects of nicotine on the nucleus accumbens and similarity to those of addictive drugs. Nature 1996;382:255-57.

49 Glassman AH. Cigarette-smoking: implications for psychiatric-illness. Am J Psychiatry 1993;150:546-53.

50 Ware JH, Dockery DW, Spiro A, et al. Passive smoking, gas cooking, and respiratory health of children living in 6 cities. Am Rev Respir Dis 1984;129:366-74.
417 
epidemiological research, followed by $16 \%$ in the field of psychology, and $12 \%$ in medicine and neuropsychopharmacology, respectively.

Table 2 lists the 100 most cited individuals 1994-2004 by total citations and the honour of the most cited author goes to an Australian, Graham Colditz, Professor in the Department of Epidemiology, Channing Laboratory, Harvard School of Public Health. The person with the highest mean number of citations is again Paul Ridker. Sixty four per cent of authors are from North America, 34\% from Europe, and 2\% from Asia. Female author representation is $22 \%$ and male authorship is $78 \%$. Fifty per cent of the authors conduct epidemiological research, and other predominant fields include branches of medicine (17\%) and psychology (13\%).

There were 53 authors who appeared on both lists.

The papers that were selected for inclusion in the top 50 (table 3) were published from 1980 to 1999, with 52\% published from 1990. Eighty per cent of the papers selected for inclusion were published in journals with ISI impact factors ranging from 2.352 (European Journal of Pharmacology) to 34.833 (New England Journal of Medicine); 12\% of the papers were published in JAMA, followed by $10 \%$ in both the Lancet (impact factor 18.316) and the New England Journal of Medicine (impact factor 34.833).

\section{DISCUSSION}

Both lists of highly cited authors are heavily dominated by "big" epidemiology papers, often being multiple risk factor studies where tobacco was one (important) variable being examined. These studies have often been the bedrock on which tobacco control policy rests and so are deservedly important. More practically, they are often cited in the introductory sections of papers when authors often run through a summary of why smoking is a health problem. Similar observations apply to another heavily cited category: nicotine pharmacology.

There are several reasons to limit interpretations of the results presented. Firstly, we recognise that use of WoS for citation analysis has been widely criticised, ${ }^{6}$ and as WoS does not cover all papers published the results should only be considered as "ball park" figures. Secondly, as a research study it is not precisely reproducible because: (1) WoS is a dynamic database, updated on a weekly basis; (2) authors selected for inclusion may differ between reviewers; and (3) it is impossible to ensure that all papers for an author have been detected due to potential errors in the data as well as finding all works of authors using two or more names. Thirdly, the selection criteria for including authors $(\geqslant 10$ publications as first author) may have excluded authors who had highly cited papers. We imposed this threshold to reduce the number of individuals who assisted in the preparation of frequently cited papers but did not make the most significant contribution. Fourthly, as the preliminary data involved over 100000 records and were merged in batches there is a probability that an author may have been excluded from the final list of names due to computation errors. Further limitations include the assumption that many authors try to support the interpretation of their own results when attempting to convince or persuade readers. ${ }^{8}$ Biased citing, such as self citation and bias towards citing English language publications, are recognised as potential problems. ${ }^{4}$ A study of citation counts in the area of addiction found that the number of citations received by papers appeared to reflect the geographic region of the study (the huge dominance of American authors citing American journals) rather than necessarily their importance as agreed by peers. ${ }^{9}$ These criticisms and observations should be taken into consideration when interpreting the results.

\section{Authors' affiliations}

F Byrne, S Chapman, School of Public Health, University of Sydney, NSW, Australia

Competing interests: none declared

Correspondence to: Professor Simon Chapman, School of Public Health, Edward Ford building A27, University of Sydney, NSW 2006, Australia; simonchapman@health.usyd.edu.au

\section{REFERENCES}

1 Marx W, Schier H, Wanitschek M. Citation analysis using online databases: feasibilities and shortcomings. Scientometrics 2001:52:59-82.

2 Adams $A B$, Simonson D. Publications, citations, and impact factors of leading investigators in critical care medicine. Respir Care 2004;49:276-81.

3 Baltussen A, Kindler $\mathrm{CH}$. Citation classics in anesthetic journals. Anesth Analg 2004:98:443-51.

4 Dumont JE. The bias of citations. Trends in Biochem Sci 1989;14:327-8.

5 Smith R. Measuring the social impact of research. BMJ 2001;323:528.

6 Adam D. Citation analysis: the counting house. Nature 2002;415:726-29.

7 Garfield E. Historiographic mapping of knowledge domains literature. Journal of Information Science 2004;30:119-45.

8 Cole S. Citations and the evaluation of individual scientists. Trends Biochem Sci 1989;14:9-13.

9 West R, Mcllwaine A. What do citation counts count for in the field of addiction?: an empirical evaluation of citation counts and their link with peer ratings of quality. Addiction 2002;97:501-4. 